Cahiers de la recherche sur les droits fondamentaux

Cahiers sur les Droits Fondamentaux

11 | 2013

Le droit de la famille en (r)évolutions

\title{
La Convention du Conseil de l'Europe sur la prévention et la lutte contre la violence à l'égard des femmes et la violence domestique
}

Mamoud Zani

\section{OpenEdition}

Journals

Édition électronique

URL : https://journals.openedition.org/crdf/4650

DOI : $10.4000 /$ crdf. 4650

ISSN : 2264-1246

Éditeur

Presses universitaires de Caen

Édition imprimée

Date de publication : 1 novembre 2013

Pagination : 55-62

ISSN : 1634-8842

Référence électronique

Mamoud Zani, «La Convention du Conseil de l'Europe sur la prévention et la lutte contre la violence à l'égard des femmes et la violence domestique ", Cahiers de la recherche sur les droits fondamentaux [En ligne], 11 | 2013, mis en ligne le 01 décembre 2014, consulté le 14 novembre 2022. URL : http:// journals.openedition.org/crdf/4650; DOI : https://doi.org/10.4000/crdf.4650 


\title{
La Convention du Conseil de l'Europe sur la prévention et la lutte contre la violence à l'égard des femmes et la violence domestique
}

\author{
Mamoud ZANI \\ Maître de conférences en droit public, directeur du Centre de droit international et européen (CDIE) de I'Université de Tunis (Tunisie)
}

I. Les traits intrinsèques de la convention

A. Les principes fondamentaux

1. Le principe de prévention

2. Le principe de protection

3. Le principe de poursuites

B. La criminalisation de la violence

II. La mise en œuvre effective de la convention
A. La multiplicité des mécanismes de suivi
1. Groupe d'experts sur la lutte contre la violence à l'égard des femmes et la violence domestique
2. Comité des parties et Parlements nationaux
B. De quelques critiques relatives au contenu de la convention et son mécanisme de suivi

Le Conseil de l'Europe ${ }^{1}$, organisation supranationale composée de quarante-sept États membres, repose sur trois principes cardinaux qui constituent le droit commun de ces derniers, à savoir la démocratie, les droits de l'homme et la prééminence du droit. Ces valeurs communes énoncées au préambule ${ }^{2}$ du Statut du Conseil de l'Europe ont été $a b$ initio au cœur de l'action de l'organisation qui s'est diversifiée au fil du temps pour couvrir des questions d'une importance majeure pour la société civile et la famille, notamment celle de la violence à l'égard des femmes, phénomène planétaire et sujet d'une actualité brûlante en droit européen de la famille.

La violence à l'égard des femmes entrave sérieusement lesdites valeurs, d'où l'intérêt porté par le Conseil de l'Europe à cette question dans le droit fil des initiatives entreprises par l'Organisation des Nations unies ${ }^{3}$ pour lutter contre toutes les formes de violence faites aux femmes. Effectivement, depuis 1990, le Comité directeur

1. Il a été créé le 5 mai 1949 par dix États membres: Belgique, Danemark, France, Irlande, Italie, Luxembourg, Pays-Bas, Norvège, Suède, RoyaumeUni de Grande-Bretagne et d'Irlande du Nord. Son siège permanent est à Strasbourg (France).

2. Son paragraphe 3 souligne l'attachement des États membres aux «valeurs spirituelles et morales qui sont le patrimoine commun de leurs peuples et qui sont à l'origine des principes de liberté individuelle, de liberté politique et de prééminence du droit, sur lesquels se fonde toute démocratie véritable».

3. Voir la Convention des Nations unies sur l'élimination de toutes les formes de discrimination à l'égard des femmes (1979), la Recommandation générale $\mathrm{n}^{\circ} 19$ (1992) du Comité pour l'élimination de la discrimination à l'égard des femmes sur la violence à l'égard des femmes et la Déclaration de l'Assemblée générale des Nations unies sur l'élimination de la violence à l'égard des femmes (1993). 
de cette institution pour l'égalité entre les femmes et les hommes (CDEG) a lancé une série d'initiatives pour promouvoir la protection des femmes contre la violence. À l'occasion de la $3{ }^{\mathrm{e}}$ Conférence ministérielle européenne sur l'égalité entre les femmes et les hommes, un plan ${ }^{4}$ d'action fut adopté pour renforcer davantage cette lutte. Il a été complété par la suite par des stratégies pour l'élimination de la violence à l'égard des femmes dans la société.

Sur le plan normatif, le salut en matière de prévention et de lutte contre la violence à l'égard des femmes et la violence domestique est venu du Comité des ministres du Conseil de l'Europe 5 avec l'adoption en 2002 de la Recommandation Rec (2002) 5 sur la protection des femmes contre la violence ${ }^{6}$. Cet instrument de "droit $^{2}$ mou» ou "fragile» (soft law) a constitué un tournant décisif dans la lutte contre la violence à l'égard des femmes: une politique globale de prévention de la violence et de protection des victimes a vu le jour dans l'ensemble des États membres du Conseil de l'Europe. Plus largement et de manière significative, ladite recommandation réaffirme deux aspects importants :

[...] la violence à l'égard des femmes découle de rapports de force inégaux entre hommes et femmes, et aboutit à une grave discrimination envers le sexe féminin tant au sein de la société que de la famille?

[...] la violence à l'égard des femmes va à l'encontre de l'instauration de l'égalité et de la paix, et constitue un obstacle majeur pour la sécurité des citoyens et la démocratie en Europe ${ }^{8}$.

Dans une optique similaire, il sied de mentionner les résolutions et recommandations adoptées tant par l'Assemblée parlementaire que par le Congrès des pouvoirs locaux et régionaux ${ }^{9}$ du Conseil de l'Europe se rapportant aux diverses formes de violence à l'égard des femmes. Il en est ainsi pour le premier organe des résolutions 1247 (2001) sur les mutilations sexuelles féminines et 1582 (2002) sur la violence domestique, ainsi que les recommandations 1723 (2005) sur les mariages forcés et les mariages d'enfants et 1777 (2007) sur les agressions sexuelles liées aux «drogues du viol». Quant au second organe, on peut relever la résolution 279 (2009) et la recommandation 260 (2009) intitulées «Combattre la violence domestique à l'égard des femmes » dont les rédacteurs de la Convention sur la prévention et la lutte contre la violence à l'égard des femmes et la violence domestique se sont inspirés.

Dans le but de consolider plus en avant la politique envisagée par la Recommandation Rec (2002) 5, et partant, d'éradiquer in globo le phénomène de la violence à l'égard des femmes, les États membres du Conseil de l'Europe ont plaidé à l'occasion du Sommet de Varsovie, tenu en Pologne les 16 et 17 mai 2005, pour une campagne mondiale ${ }^{10}$ contre la violence et une Task Force ${ }^{11}$ du Conseil de l'Europe pour combattre la violence à l'égard des femmes, y compris la violence domestique, a été mise en place en vue de supputer les mesures nationales et internationales visant la lutte contre ce phénomène planétaire. Cette évaluation a permis de pointer du doigt les insuffisances des mesures préconisées tout en recommandant l'élaboration par l'institution strasbourgeoise d'une convention contraignante destinée à prévenir et à combattre la violence à l'égard des femmes.

Force est de remarquer que les ministres européens de la Justice ont également appelé, lors de leur $27^{\mathrm{e}}$ conférence tenue en 2006 à Erevan (Arménie), à l'adoption d'un instrument juridique contraignant sur la question; de même que l'Assemblée parlementaire du Conseil de l'Europe par le truchement de sa résolution 1635 (2008) et de sa recommandation 1847 (2008). L'intitulé de ces instruments parle de lui-même: «Combattre la violence à l'égard des femmes, pour une convention du Conseil de l'Europe».

Pour examiner de manière approfondie la question d'une convention sur le sujet, le Comité des ministres a institué, en 2008, un groupe d'experts appelé Comité ad hoc pour prévenir et combattre la violence à l'égard des femmes et la violence domestique (CAHVIO), afin de rédiger un projet de convention

[...] pour prévenir et combattre la violence domestique, y compris les formes spécifiques de violence à l'égard des femmes, d'autres formes de violence à l'égard des femmes, et pour protéger et soutenir les victimes de tels actes de violence et poursuivre leurs auteurs ${ }^{12}$.

4. Voir le rapport du Groupe de spécialistes pour la lutte contre la violence à l'égard des femmes (EG-S-VL), Conseil de l'Europe, Strasbourg, février 1997.

5. Organe de décision du Conseil de l'Europe, il est composé des ministres des Affaires étrangères des 47 États membres.

6. Adoptée le 30 avril 2002, lors de la $794^{\mathrm{e}}$ réunion des délégués des ministres. Voir C. Hagemann-White, Protéger les femmes contre la violence. Étude analytique des résultats du troisième cycle de suivi de la mise en cuvre de la Recommandation Rec (2002) 5 sur la protection des femmes contre la violence dans les États membres du Conseil de l'Europe, Direction générale des droits de l'homme et des affaires juridiques, Conseil de l'Europe, Strasbourg, 2010

7. Comité des ministres du Conseil de l’Europe, Recommandation Rec (2002) 5, préambule, $\$ 2$.

8. Ibid., $\$ 6$.

9. C’est une assemblée politique paneuropéenne composée de 636 élus (conseillers régionaux et municipaux, maires et présidents de région) représentant plus de 200000 collectivités de 47 pays européens.

10. Cette campagne a été menée entre 2006 et 2008 . Voir les actes de la Conférence de clôture de la campagne du Conseil de l'Europe pour combattre la violence à l'égard des femmes, y compris la violence domestique, EG-VAW-CONF (2008) 1, Direction générale des droits de l'homme et des affaires juridiques, Conseil de l'Europe, Strasbourg, 10-11 juin 2008.

11. Voir Task Force to Combat Violence against Women, including Domestic Violence (EG-TFV), Final Activity Report, Conseil de l'Europe, Strasbourg, 2008.

12. Voir le rapport explicatif du Comité des ministres sur la convention, STCE nº 210, Conseil de l’Europe, Strasbourg, avril 2011, p. 4, $\$ 19$. 
À la suite d'une série de négociations engagée depuis 2009 avec les États parties du Conseil de l'Europe, le CAHVIO a achevé le texte du projet de convention en 2010 et l'Assemblée parlementaire a rendu, le 11 mars 2013, un avis favorable sur ledit projet. En définitive, la Convention sur la prévention et la lutte contre la violence à l'égard des femmes et la violence domestique a été adoptée par le Comité des ministres, le 7 avril 2011, et est ouverte largement à la signature « des États membres du Conseil de l'Europe, des États non membres ${ }^{13}$ ayant participé à son élaboration ainsi que de l'Union européenne " ${ }^{14}$, le 11 mai 2011.

La Convention d'Istanbul (lieu de son adoption) qui n'est pas encore en vigueur, faute du nombre ${ }^{15} \mathrm{de}$ ratifications requis par l'article 75, alinéa 3, vient prima facie conforter à coup sûr le corpus normatif souple dont dispose le Conseil de l'Europe en matière de prévention et de lutte contre la violence à l'égard des femmes et la violence domestique. C'est un instrument révolutionnaire de valeur juridique obligatoire dont la particularité consiste à consolider considérablement l'action du Conseil dans ledit domaine; d'où l'intérêt d'examiner sa valeur ajoutée à travers la problématique suivante: quelles sont la portée et les limites de la Convention sur la prévention et la lutte contre la violence à l'égard des femmes et la violence domestique? Pour ce faire, nous examinerons, tout d'abord, les traits intrinsèques de la convention (I), ensuite, la mise en œuvre effective de cet instrument (II).

\section{Les traits intrinsèques de la convention}

La Convention du Conseil de l'Europe sur la prévention et la lutte contre la violence à l'égard des femmes et la violence domestique comprend douze chapitres, chacun d'eux porte sur une question spécifique. Cette spécificité permet de cibler les principes fondamentaux (A) sur lesquels repose la convention, ainsi que la criminalisation de nombreuses formes de violence (B).

\section{A. Les principes fondamentaux}

L'objectif principal de la convention consiste suivant le paragraphe 17 de son préambule, à instituer « une Europe libre de violence à l'égard des femmes et de violence domestique». Pour atteindre cet objectif, la convention précise que la concrétisation de l'égalité entre les femmes ${ }^{16}$ et les hommes constitue, «un élément clé dans la prévention de la violence à l'égard des femmes ${ }^{17}$. Cette violence de nature double - violence à l'égard des femmes et violence domestique - atteste

[...] des rapports de force historiquement inégaux entre les femmes et les hommes ayant conduit à la domination et à la discrimination des femmes par les hommes, privant ainsi les femmes de leur pleine émancipation ${ }^{18}$.

La notion de violence est définie de manière large par l'article 3 de la convention en tenant compte de ses diverses formes; ainsi la "violence à l'égard des femmes ${ }^{19}$ est considérée comme une violation des droits de l'homme et une forme de discrimination; la "violence domestique» couvre pour sa part

[...] tous les actes de violence physique, sexuelle, psychologique ou économique qui surviennent au sein de la famille ou du foyer ou entre des anciens ou actuels conjoints ou partenaires, indépendamment du fait que l'auteur de

l'infraction partage ou a partagé le même domicile que la victime ${ }^{20}$.

Quant à la «violence à l'égard des femmes fondée sur le genre $»^{21}$, celle-ci concerne toute violence faite à une femme en tant que telle (une femme), ou affectant les femmes de manière disproportionnée. Cette forme de violence est assez particulière par rapport aux autres formes de violence du fait que «la nature structurelle de la violence à l'égard des femmes est fondée sur le genre $»^{22}$, c'est-à-dire «les rôles, les comportements, les activités et les attributions socialement construits, qu'une société donnée considère comme appropriés pour les femmes et les hommes ${ }^{23}$.

13. Il s'agit du Canada, du Saint-Siège, du Japon, du Mexique et des États-Unis d'Amérique.

14. Convention sur la prévention et la lutte contre la violence à l'égard des femmes et la violence domestique, art. 75, al. 1 .

15. Dix ratifications sont nécessaires pour l'entrée en vigueur de cet instrument. Au 30 mars 2013, la convention enregistrait vingt-six signatures et trois ratifications (Albanie, Portugal et Turquie).

16. La notion de «femme» inclut, suivant l'alinéa f de l'article 3, toutes les filles de moins de 18 ans.

17. Paragraphe 9 du préambule.

18. Paragraphe 10 du préambule.

19. Art. 3, al. a. Dans sa résolution 11/2 du 17 juin 2009 intitulée «Intensification de l'action menée pour éliminer toutes formes de violence à l'égard des femmes», le Conseil des droits de l'homme des Nations unies a défini la «violence à l'égard des femmes» comme étant tout «acte de violence sexiste qui cause, ou est susceptible de causer à la femme une atteinte à son intégrité ou une souffrance physique, sexuelle ou psychologique, y compris la menace d'un tel acte, la contrainte et la privation arbitraire de liberté, que ce soit dans la vie publique ou dans la vie privée» ( $\$ 1)$. Cette définition est reprise par la Commission de la condition de la femme, dans son projet de conclusions de mars 2013 ( $57^{\mathrm{e}}$ session), sur «l'élimination et la prévention de toutes les formes de violence à l'égard des femmes et des filles»; doc. E/CN.6/2013.L.5, 19 mars 2013, p. $2-3, \S 11$.

20. Art. 3, al. b.

21. Art. 3, al. d.

22. Paragraphe 11 du préambule. Il faut remarquer que les rédacteurs de la convention ont considéré le critère de «genre» comme une sorte de baromètre pour évaluer l'application des dispositions de la convention par les États parties, d'où l'article 6 affirmant que: «Les Parties s'engagent à inclure une perspective de genre dans la mise en œuvre et l'évaluation de l'impact des dispositions de la présente convention et à promouvoir et mettre en œuvre de manière effective des politiques d'égalité entre les femmes et les hommes, et d'autonomisation des femmes».

23. Art. 3, al. c. 
Pour éradiquer l'ensemble des formes de violence décrit ci-dessus, la Convention sur la prévention et la lutte contre la violence à l'égard des femmes et la violence domestique préconise trois principes fondamentaux qui font peser sur les États parties des obligations juridiques, obligations $^{24}$ à la fois de moyens et de résultat, afin de prévenir la violence, d'en protéger les victimes et de sanctionner leurs auteurs. Le jargon strasbourgeois utilise le vocabulaire ou la doctrine des trois $\mathrm{P}$ pour désigner les principes décrits ci-après.

\section{Le principe de prévention}

La prévention de la violence à l'égard des femmes et de la violence domestique est envisagée par le chapitre III de la convention. Celui-ci comprend un article clé, en l'occurrence l'article 12 relatif aux obligations générales des États parties en matière de prévention, ainsi que les mesures nécessaires de sensibilisation, d'éducation et de formation que lesdits États devraient prendre.

Dans le cadre des mesures générales de prévention, les États parties sont tenus de promouvoir les changements de mentalité et de comportement afin

[...] d'éradiquer les préjugés, les coutumes, les traditions et toute autre pratique fondés sur l'idée de l'infériorité des femmes ou sur un rôle stéréotypé des femmes et des hommes ${ }^{25}$.

Ils doivent également prendre des mesures législatives pour prévenir toutes les formes de violence couvertes par le champ d'application de la convention commises par toute personne physique ou morale, tout en tenant compte des besoins spécifiques des personnes vulnérables ${ }^{26}$; ainsi que pour assurer la promotion de programmes et d'activités visant l'autonomisation des femmes sur les plans politique et économique. Au surplus, ils doivent veiller à ce que

[...] la culture, la coutume, la religion, la tradition ou le prétendu «honneur» ne soient pas considérés comme justifiant des actes de violence couverts par le champ d'application de la convention ${ }^{27}$.

Pour ce qui est des autres mesures, les États parties doivent ${ }^{28}$ globalement informer le grand public des différentes formes de violence affectant les femmes, promouvoir l'égalité entre les femmes et les hommes, former les professionnels aux causes de toutes les formes de violence, soutenir les programmes en faveur des auteurs de violence domestique, encourager le secteur privé et les médias à prendre part à l'élaboration et la mise en œuvre des politiques visant la prévention de la violence à l'égard des femmes.

\section{Le principe de protection}

La protection des victimes contre tout nouvel acte de violence est couverte par les dispositions de l'article 18 de la convention. À cet effet, les États parties sont tenus de prendre au niveau interne les mesures législatives nécessaires visant la mise en place de mécanismes adéquats pour garantir une coopération effective entre les agences étatiques pertinentes (les autorités judiciaires, les procureurs, les services répressifs, les autorités locales et régionales, les organisations non gouvernementales), dans la protection et le soutien des victimes et des témoins de toutes les formes de violence. Ces mesures doivent reposer sur une compréhension fondée sur le genre de la violence à l'égard des femmes et de la violence domestique, et sur une approche intégrée qui tient suffisamment compte des relations entre les victimes, les auteurs des infractions, les enfants et leur environnement social plus large ${ }^{29}$.

La protection s'étend à d'autres domaines que les États parties doivent couvrir par d'autres mesures complémentaires: l'information des victimes sur les divers services de soutien et les mesures juridiques dont elles disposent; la garantie de l'accès des victimes aux services facilitant leur rétablissement (conseil juridique et psychologique, assistance financière, logement, éducation) et aux services de santé et sociaux; le soutien aux victimes en matière de plaintes individuelles / collectives devant les mécanismes régionaux et internationaux; la mise en place de centres d'aide d'urgence accessibles pour les victimes de viols et de violences sexuelles et, surtout, la prise en compte des droits et besoins des enfants témoins de toutes les formes de violence.

\section{Le principe de poursuites}

En vertu de l'article 49 de la convention, les États parties ont l'obligation de veiller à ce que les enquêtes et les procédures judiciaires relatives à toutes les formes de violence soient traitées de manière prompte et sans aucun retard injustifié. Ces enquêtes et procédures doivent se conformer aux principes fondamentaux des droits de l'homme et tiennent compte de la compréhension de la violence fondée sur le genre pour l'efficacité de ces démarches.

24. Voir l'art. 5 de la convention («Obligations de l'État et diligence voulue»). En ce sens, voir aussi J. Combacau, «Obligations de résultat et obligations de comportement - Quelques questions et pas de réponse », in Le droit international, unité et diversité: mélanges offerts à Paul Reuter, Paris, A. Pedone, 1981, p. 181-204.

25. Art. 12, al. 1

26. Il s'agit des femmes enceintes, des mères célibataires et des personnes handicapées.

27. Art. 12, al. 5 .

28. Voir les art. 13 à 17.

29. Voir l'art. 18, al. 3. 
La garantie des preuves ayant trait aux antécédents sexuels et à la conduite de la victime est envisagée par l'article 54 de la convention. À cet égard, les États parties doivent privilégier, dans les procédures civiles ou pénales, les preuves uniquement de nature évidente, c'està-dire celles qui sont pertinentes et nécessaires. Quant aux enquêtes et poursuites se rapportant à un certain nombre de catégories d'infractions (violence physique, violence sexuelle, y compris le viol, mutilations génitales féminines, avortement et stérilisation forcés) ${ }^{30}$, les États parties veillent à ce que celles-ci ne relèvent pas complètement d'une dénonciation ou d'une plainte de la victime, et qu'en définitive toute procédure engagée puisse se poursuivre même si la victime se rétracte ou retire sa plainte. Au cours de l'enquête et de la procédure judiciaire, les victimes peuvent bénéficier de l'appui des organisations gouvernementales et non gouvernementales et des conseillers spécialisés dans la violence domestique ${ }^{31}$.

À tous les stades des enquêtes et des procédures judiciaires, les États parties sont tenus de prendre un certain nombre de mesures ${ }^{32}$ pour protéger les victimes de toutes les formes de violence: il en va ainsi de la protection des victimes des risques d'intimidation, de représailles et de nouvelle victimisation; l'information des victimes en cas d'évasion de l'auteur de l'infraction ou de sa libération temporaire ou définitive; la préservation des droits et intérêts des victimes et la mise à disposition des interprètes indépendants et compétents; la possibilité pour les victimes de témoigner en salle d'audience sans être présentes, ou du moins sans que l'auteur présumé de l'infraction ne soit présent. Une attention particulière est conférée aux enfants victimes et témoins de violence à l'égard des femmes et de violence domestique.

Tenant compte de la situation des enfants victimes, l'article 58 de la convention prévoit pour l'effectivité des poursuites judiciaires, dans le cas des infractions citées ci-dessus, l'allongement du délai de prescription pour une durée suffisante et proportionnelle à la gravité de l'infraction commise.

\section{B. La criminalisation de la violence}

La criminalisation de certaines infractions, en particulier celles mentionnées dans les articles 33 à 39 de la convention, constitue la valeur ajoutée certaine de l'instrument européen. En effet, ces dispositions exigent des États parties d'ériger en infraction pénale la violence psychologique, celle-ci étant définie comme une conduite intentionnelle qui porte "gravement atteinte à l'intégrité psychologique d'une autre personne par la contrainte ou les menaces ${ }^{33}$. Il en est de même pour le harcèlement ${ }^{34}$, une conduite intentionnelle d'une personne adoptant, à plusieurs reprises, un comportement menaçant dirigé vers une autre personne au point qu'elle craint pour sa sécurité. Et aussi la violence physique, un acte intentionnel commis à l'égard d'une autre personne ${ }^{35}$.

Dans le même ordre d'idées, la violence sexuelle, y compris le viol doivent être incriminés par les États parties. L'article 36 de la convention comprend les formes d'actes sexuels suivantes:

a. la pénétration vaginale, anale ou orale non consentie, à caractère sexuel, du corps d'autrui avec toute partie du corps ou avec un objet;

b. les autres actes à caractère sexuel non consentis sur autrui;

c. le fait de contraindre autrui à se livrer à des actes à caractère sexuel non consentis ${ }^{36}$ avec un tiers.

Il s'étend aussi aux «actes commis contre les anciens ou actuels conjoints ou partenaires ${ }^{37}$.

S'agissant des mariages forcés ${ }^{38}$, les États parties sont appelés à incriminer deux sortes de conduites: le fait de forcer une personne à contracter un mariage et le fait de tromper une personne à l'étranger dans le but de la forcer à contracter un mariage. Pour l'infraction pénale de mutilation génitale féminine, l'article 38 de la convention vise de manière précise les actes suivants : l'excision, l'infibulation ou toute autre mutilation de la totalité ou partie des labia majora, labia minora ou clitoris d'une femme; le fait de contraindre une femme à subir l'un de ces actes ou de lui fournir les moyens à cette fin; le fait d'inciter ou de contraindre une fille à subir les mêmes actes ou de lui fournir les moyens à cette fin.

D'autres actes intentionnels susceptibles d'affecter la capacité reproductive naturelle des femmes sont prévus par l'article 39 de la convention: il s'agit de l'avortement et de la stérilisation forcés. Ainsi, les États parties doivent incriminer l'avortement pratiqué sans le consentement préalable et éclairé de la victime, et la stérilisation forcée d'une femme ou d'une fille consistant à pratiquer une intervention chirurgicale afin de lui ôter la capacité de se

30. Voir les art. $35,36,37,38$ et 39 .

31. Art. 55, al. 2 .

32. Art. 56, al. a, b, c, d, e, f, g, h et i. On peut ajouter, suivant l'article 57, le droit à l'assistance d'un défenseur et à une aide juridictionnelle gratuite pour les victimes.

33. Art. 33

34. Art. 34 .

35. Art. 35

36. L'alinéa 2 de l'article 36 précise que: «Le consentement doit être donné volontairement comme résultat de la volonté libre de la personne considérée dans le contexte des circonstances environnantes».

37. Art. 36 , al. 3 .

38. Art. 37, al. 1 et 2. Voir aussi R.-A. Edwige, Les mariages forcés dans les États membres du Conseil de l'Europe, législation comparée et actions politiques, Direction générale des droits de l'homme, Conseil de l'Europe, Strasbourg, 2005. 
reproduire naturellement, sans que celle-ci n'ait donné au préalable son consentement éclairé. Par ailleurs, tout harcèlement sexuel doit faire l'objet d'une sanction pénale; l'article 40 de la convention fait allusion à trois principales formes de comportement de nature sexuelle, en l'occurrence verbal, non verbal et physique. Ces actes ${ }^{39}$ doivent avoir pour objectif ou pour effet de violer la dignité de la victime; c'est le cas, par exemple, lorsque l'acte en question génère un environnement intimidant, hostile, dégradant, humiliant ou offensant.

En vertu de l'article 41, alinéa 1 de la convention, les États parties sont tenus d'ériger en infraction pénale tout acte de complicité, en vue de commettre l'une des infractions couvertes par les articles 33 à 39 de la convention. Toutefois, en raison de la diversité des systèmes juridiques nationaux, ils ne doivent ériger en infraction pénale que les tentatives d'infractions ayant trait aux cas graves de violence physique, violence sexuelle y compris le viol, mariages forcés, mutilations génitales féminines, avortement et stérilisation forcés.

La compétence en matière d'infractions pénales relève de la responsabilité des États parties. Elle repose sur un certain nombre de principes ou critères énoncés à l'article 44 de la convention: le principe de territorialité, le principe de nationalité et le principe aut dedere aut judicare (extrader ou poursuivre). L'alinéa 3 de cet article, qui constitue à n'en point douter une valeur ajoutée dans le dispositif de la convention pour lutter de facto contre les diverses formes de violence, évacue, à juste titre, s'agissant des infractions les plus graves visées par la convention, le principe de la double incrimination selon lequel l'infraction pénale commise dans un pays donné doit avoir une pareille qualification dans le pays où elle a été commise.

L'analyse des principales caractéristiques de la Convention sur la prévention et la lutte contre la violence à l'égard des femmes et la violence domestique permet de confirmer sa valeur ajoutée certaine, mais qu'en est-il in concreto de sa mise en œuvre?

\section{La mise en œuvre effective de la convention}

La Convention sur la prévention et la lutte contre la violence à l'égard des femmes et la violence domestique préconise des mécanismes multiples pour en assurer l'application (A). La multiplicité des organes de suivi et l'appréciation de la convention sur le plan substantiel permettent de formuler quelques critiques de fond sur ces deux aspects (B).

\section{A. La multiplicité des mécanismes de suivi}

La mise en œuvre de la convention est régie par le chapitre IX. Celui-ci prévoit les mécanismes de contrôle décrits ci-après.

\section{Groupe d'experts sur la lutte contre la violence à l'égard des femmes et la violence domestique}

C’est le principal mécanisme de mise en œuvre des dispositions de la convention. En ratifiant cette dernière, les États parties se sont engagés à conformer leurs ordres juridiques nationaux respectifs avec les principes de l'instrument européen. À cet effet, ils sont tenus de mettre en place des politiques nationales ${ }^{40}$ effectives, globales et coordonnées, pour prévenir et combattre toutes les formes de violence couvertes par la convention et offrir une réponse générale à la violence à l'égard des femmes.

Conformément au paragraphe 2 de l'article 66 de la convention, le Groupe d'experts sur la lutte contre la violence à l'égard des femmes et la violence domestique (ci-après dénommé «GREVIO») est composé de dix membres au minimum et de quinze membres au maximum ressortissants des États parties, siégeant à titre individuel, indépendants et impartiaux. Ils sont élus ${ }^{41}$ selon une procédure fixée par le Comité des ministres du Conseil de l'Europe par le Comité des parties pour un mandat de quatre ans, renouvelable une seule fois. Ces membres sont choisis parmi

[...] des personnalités de haute moralité connues pour leur compétence en matière de droits de l'homme, d'égalité entre les femmes et les hommes, de violence à l'égard des femmes et de violence domestique ou d'assistance et protection des victimes, ou ayant une expérience professionnelle reconnue dans les domaines couverts par la présente convention ${ }^{42}$.

La fonction de contrôle du GREVIO repose pour l'essentiel sur l'analyse des rapports étatiques pour juger de l'efficacité des mesures d'ordre législatif et autres prises par les États parties sur le plan national, pour donner effet aux dispositions de la convention. Cette analyse peut englober aussi les informations provenant des organisations non gouvernementales et de la société civile, ainsi que des institutions nationales de protection des droits de l'homme ${ }^{43}$. En cas de violations graves et systématiques des dispositions de la convention, le GREVIO peut exiger la soumission urgente d'un rapport spécial relatif aux mesures prises pour prévenir un type de violence grave, répandu ou récurrent à l'égard des femmes.

À côté de la technique des rapports, il existe le système des visites à l'instar du Comité européen de prévention de

39. Il convient de préciser que ces actes doivent être imposés à la victime par l'auteur de l'infraction.

40. Art. 7 de la convention.

41. Suivant l'art. 66, al. 3, "l'élection initiale de 10 membres est organisée dans un délai d'un an suivant la date d'entrée en vigueur de la présente convention. L'élection de cinq membres additionnels est organisée après la vingt-cinquième ratification ou adhésion ».

42. Art. 66, al. 4 (a).

43. Voir l'art. 68, al. 5. Ces informations peuvent également émaner du commissaire aux droits de l'homme du Conseil de l'Europe, de l'Assemblée parlementaire et d'autres organes spécialisés pertinents du Conseil de l'Europe ainsi que ceux établis par d'autres instruments internationaux. 
la torture, envisagé par l'article 66, alinéa 7 de la convention. En effet, en cas d'un rapport jugé insuffisant par le GREVIO, ce dernier peut, en coopération avec les autorités nationales et l'assistance d'experts nationaux indépendants, organiser des visites dans les pays concernés. Il peut même désigner un ou plusieurs de ses membres pour conduire une enquête ${ }^{44}$ et lui présenter un rapport d'urgence. Une fois que les conclusions de l'enquête lui sont parvenues, le GREVIO les transmet à son tour à l'État partie concerné et, le cas échéant, au Comité des parties et au Comité des ministres du Conseil de l'Europe en y ajoutant ses propres commentaires et recommandations. Sur la base de celles-ci, le Comité des parties peut adresser à l'État partie défaillant des recommandations concernant les mesures à prendre pour mettre en œuvre les conclusions du GREVIO, tout en fixant une date pour la réception du complément d'information demandé sur la mise en œuvre.

\section{Comité des parties et Parlements nationaux}

Le Comité des parties ${ }^{45}$ et les Parlements nationaux exercent, à vrai dire, un contrôle subsidiaire par rapport à celui du GREVIO. Le Comité des parties, organe politique, comprend principalement les États parties à la convention. Il sera convoqué pour la première fois par le Secrétaire général du Conseil de l'Europe, dans un délai d'un an suivant l'entrée en vigueur de la convention, afin d'élire les membres du GREVIO. Il se réunira par la suite à la demande d'un tiers des parties, du Secrétaire général du Conseil de l'Europe ou du président du GREVIO. L'instance en question permet de conforter la coopération avec ce dernier en vue d'une mise en œuvre concrète de la convention.

À leur tour, les Parlements nationaux se voient associés dans le processus de mise en œuvre de la convention dans les ordres juridiques nationaux. Au demeurant, les États parties ont l'obligation, suivant l'article 70 de la convention, d'inviter les Parlements nationaux à participer au suivi et de leur soumettre les rapports du GREVIO pour consultation. Pour sa part, l'Assemblée parlementaire du Conseil de l'Europe tenant compte de son action en matière de violence à l'égard des femmes est aussi impliquée dans le processus de suivi de la convention. L'alinéa 3 de l'article 70 précise de manière originale qu'elle fera de manière régulière un bilan de l'application dudit instrument.

\section{B. De quelques critiques relatives au contenu de la convention et son mécanisme de suivi}

La protection des femmes contre toutes les formes de violence est devenue une réalité avec l'adoption de la
Convention sur la prévention et la lutte contre la violence à l'égard des femmes et la violence domestique. Bien que celle-ci comble véritablement une grave lacune sur le plan normatif, un regard critique sur son contenu et le mécanisme de suivi préconisé semble démontrer la portée limitée de l'instrument européen. Ainsi, dès le départ on est frappé par la longueur de la convention qui compte quatre-vingt-un articles et une annexe consacrée aux privilèges et immunités dont bénéficient les membres du GREVIO et ceux chargés d'effectuer des visites par pays, alors que la Convention de sauvegarde des droits de l'homme et des libertés fondamentales ne compte que cinquante-neuf articles en dehors des quatorze protocoles ${ }^{46} \mathrm{~d}$ 'amendement. Ce nombre d'articles risque fortement d'atténuer la visibilité de la convention ellemême par rapport à l'objectif escompté et les obligations étatiques, d'autant plus qu'on aurait pu parfaitement faire l'économie de l'annexe, en prévoyant celle-ci, par exemple, dans le règlement intérieur du GREVIO.

Il va de soi que la convention constitue une sorte de révolution dans l'espace juridique européen à la lumière de certaines de ses dispositions ${ }^{47}$ et de sa nature obligatoire, pourtant l'analyse attentive d'un certain nombre de ses dispositions permet une lecture tout à fait opposée, $\mathrm{du}$ fait de la formulation de certains articles relevant plus du droit déclaratoire que du droit obligatoire. Les expressions suivantes contenues dans un bon nombre d'articles confirment bel et bien ce constat: «Les parties prennent les mesures législatives et autres nécessaires » - expression qui domine la totalité des dispositions de la convention; «Les parties prennent les mesures nécessaires» (art. 12 et 28); «Les parties s'efforcent d'effectuer des enquêtes» (art. 11, al. 2); «Les parties s'efforcent de prendre les mesures législatives ou autres nécessaires » (art. 44, al. 2); "Les parties s'efforcent d'intégrer, le cas échéant, la prévention et la lutte contre la violence à l'égard des femmes et la violence domestique» (art. 62, al. 4).

Dans un contexte similaire, il convient de préciser que la formulation de certains articles est également maladroite et confère aux États parties un large pouvoir d'appréciation quant aux mesures nationales à prendre pour l'application de la convention. C'est le cas, par exemple, du membre de phrase: les «membres du GREVIO [...] se rendent disponibles pour remplir leurs fonctions de manière effective» (art. 66, al. 4, e); et de l'article 40 in fine laissant aux États parties le choix de traiter le harcèlement sexuel comme une infraction pénale ou de lui appliquer d'autres sanctions légales. Au reste, l'article 78 aurait dû préciser qu'aucune réserve ${ }^{48}$ incompatible avec l'objet et le but de la convention ne sera admise, au lieu de l'expression vague

44. Elle nécessite l'aval de l'État partie concerné et peut aboutir à une visite sur son territoire.

45. Art. 67.

46. Le Protocole $\mathrm{n}^{\circ} 14$ à la Cour EDH amendant le système de contrôle de la convention est entré en vigueur le $1^{\mathrm{er}}$ juin 2010 . Voir aussi l'accord de Madrid du 12 mai 2009.

47. Par exemple, l'indemnisation des victimes de violences (art. 30).

48. L'Allemagne, Malte, la Pologne et la Serbie ont formulé des réserves à l'encontre de certaines dispositions de la convention. 
et imprécise: «aucune réserve n'est admise à l'égard des dispositions de la présente convention» (al. 1).

L'article 75 de la convention reste muet sur les conséquences de l'adhésion des États tiers, principalement ceux qui ont été associés dans son élaboration. De plus, il conditionne son entrée en vigueur à l'obtention de dix instruments de ratification, un chiffre qui semble démesuré par rapport aux objectifs de la convention. Il est d'ailleurs surprenant que l'instrument européen ne puisse être ratifié que par trois États, alors qu'il fut adopté en 2011.

S'agissant du mécanisme de suivi de la convention, l'article 68 relatif au fonctionnement de la procédure de suivi reste lacunaire sur de nombreux points : rien n'est dit sur le cas de non-coopération d'un État partie ou l'envoi tardif par les États parties de leurs rapports nationaux sur la mise en œuvre de la convention; rien n'est dit non plus sur les pouvoirs du GREVIO pour faire face à de telles situations. Certes, l'alinéa 11 de l'article en question fait allusion à la sanction morale (publication du rapport et des conclusions du GREVIO), mais ne donne aucune indication des mesures dont dispose ledit organe pour obliger l'État défaillant à respecter ses obligations, hormis l'hypothèse du recours au Comité des ministres du Conseil de l'Europe. En outre, l'article 7o, alinéa 2 de la convention demande aux États parties de transmettre les rapports du GREVIO à leurs Parlements nationaux, sans pour autant exiger d'eux une large diffusion sur le plan national. Enfin, aucune mesure n'est envisagée à l'encontre des États parties qui n'établissent pas, au sens de l'article 10, alinéa 1 de la convention, des organes nationaux de coordination et de mise en œuvre des principes de la convention au plan national.

L'œuvre du Conseil de l'Europe en matière de prévention et de lutte contre la violence sous toutes ses formes est salutaire avec l'adoption de la Convention sur la prévention et la lutte contre la violence à l'égard des femmes et la violence domestique. C'est un traité audacieux qui prend à bras-le-corps la question délicate de la violence avec sa double nature et consolide incontestablement l'arsenal normatif existant de l'institution strasbourgeoise dans le cas d'espèce, notamment à travers les trois principes fondamentaux qu'il véhicule (prévention, protection et poursuite) et la criminalisation de certaines infractions telles que la violence psychologique, la violence sexuelle, y compris le viol et les mariages forcés. Toutefois, la valeur ajoutée patente de l'instrument se fond avec les multiples expressions maladroites caractérisant un certain nombre de ses dispositions et celles-ci risqueraient sérieusement de limiter sa portée en pratique et par la même occasion affaiblir son application. Sur ce dernier point, les États parties disposent d'un pouvoir discrétionnaire assez large quant aux mesures nationales d'application, d'autant plus que la question de la coordination dans les États à structure fédérale pour la mise en ouvre des dispositions de la convention n'a pas tout à fait été traitée. Gardons tout de même une lueur d'espoir pour l'entrée en vigueur rapide de l'instrument européen afin de pouvoir changer les mentalités par rapport à la violence sexiste ${ }^{49}$ et construire une Europe sans violence à l'égard des femmes et sans violence domestique.

49. Dans ce sens, la Recommandation $n^{\circ} 19$ (1992) du Comité des Nations unies pour l'élimination de la discrimination à l'égard des femmes précise que: «la violence fondée sur le sexe est une forme de discrimination qui empêche sérieusement les femmes de jouir des droits et libertés au même titre que les hommes» (principe 1). 